\title{
Approximate Solution of the Singular-Perturbation Problem on Chebyshev-Gauss Grid
}

\author{
Mustafa Gülsu, Yalçın Öztürk \\ Department of Mathematics, Faculty of Science, Mugla University, Mugla, Turkey \\ E-mail:mgulsu@mu.edu.tr \\ Received April 27, 2011; revised May 24, 2011; accepted June 5, 2011
}

\begin{abstract}
Matrix methods, now-a-days, are playing an important role in solving the real life problems governed by ODEs and/or by PDEs. Many differential models of sciences and engineers for which the existing methodologies do not give reliable results, these methods are solving them competitively. In this work, a matrix methods is presented for approximate solution of the second-order singularly-perturbed delay differential equations. The main characteristic of this technique is that it reduces these problems to those of solving a system of algebraic equations, thus greatly simplifying the problem. The error analysis and convergence for the proposed method is introduced. Finally some experiments and their numerical solutions are given.
\end{abstract}

Keywords: Singular Perturbation Problems, Two-Point Boundary Value Problems, The Shifted Chebyshev Polynomials, Approximation Method, Matrix Method

\section{Introduction}

The boundary-value problems for singularly perturbed delay-differential equations arise in various practical problems in biomechanics and physics such as in variational problem in control theory. These problems mainly depend on a small positive parameter and a delay parameter in such a way that the solution varies rapidly in some parts of the domain and varies slowly in some other parts of the domain. Moreover, this class of problems possess boundary layers, i.e. regions of rapid change in the solution near one of the boundary points. There is a wide class of asymptotic expansion methods available for solving the above type problems. But there can be difficulties in applying these asymptotic expansion methods, such as finding the appropriate asymptotic expansions in the inner and outer regions, which are not routine exercises but require skill, insight and experimentation. The numerical treatment of singularly perturbed problems present some major computational difficulties and in recent years a large number of specialpurpose methods have been proposed to provide accurate numerical solutions [1-5] by Kadalbajoo. This type of problem has been intensively studied analytically and it is known that its solution generally has a multiscale character; i.e. it features regions called "boundary layers" where the solution varies rapidly. And these equa- tions as well as numerical methods have been studied by several authors [6-10]. The outer solution corresponds to the reduced problem, i.e., that obtained by setting the small perturbation parameter to zero. In recent years, the Chebyshev method has been used to find the approximate solutions of differential, difference, integral and integro-differential-difference equations [11,12]. The main characteristic of this technique is that it reduces these problems to those of solving a system of algebraic equations, thus greatly simplifying the problem.

Consider the of singularly-perturbed delay differential equations form

$$
\varepsilon y^{\prime \prime}(x)+p(x) y^{\prime}(x-\delta)+q(x) y(x)=g(x)
$$

where $0<x<1$, with the boundary conditions $y(0)=\alpha_{0}$, $y(1)=\alpha_{1}$ and $\varepsilon$ is a small positive parameter $0<\varepsilon \ll 1$, $\delta$ is also a small shifting parameter $0<\delta \ll 1, p(x)$, $q(x), r(x), s(x)$ and $f(x)$ are sufficiently smooth functions. Our goal is to find an approximate solution expressed polynomial of degree $N$ in the form

$$
y_{N}(x)=\sum_{r=0}^{N} a_{r} T_{r}^{*}(x)
$$

where $a_{r}$ unknown coefficients, $T_{r}^{*}(x)$ is the shifted Chebyshev polynomials of the first kind and $N$ is chosen any positive integer such that $N \leq m$. To obtained a solution (2) of the problem (1), we can use the zeroes of 
the shifted Chebyshev polynomials $T_{N+1}^{*}(x)$ of the first kind defined by

$$
x_{i-1}=\frac{1}{2}\left(1+\cos \left(\frac{\left(n-i+\frac{3}{2}\right) \pi}{N}\right)\right), i=1, \cdots, N+1
$$

\section{Basic Idea}

Polynomials are the only functions that a computer can evaluate exactly, so we make approximate functions $[a, b] \rightarrow \mathbb{R}$ by polynomials. The uniform norm (or Chebyshev norm, maximum norm) is defined by

$$
\|f\|_{\infty}=\max _{x \in[a, b]}|f(x)| \text {. }
$$

Definition 2.1. For a given continuous function $f \in C[a, b]$, a best approximation polynomial of degree $N$ is a polynomial $p_{N}^{*}(f) \in \mathbb{P}_{N}$ such that

$$
\left\|f-p_{N}^{*}(f)\right\|_{\infty}=\min \left\{\|f-p\|_{\infty}: p \in \mathbb{P}_{N}\right\}
$$

It is a good idea to approximate function by polynomials, because the classical Weierstrass Theorem is a fundamental result in the approximation of continuous functions by polynomials [13-15].

Theorem 2.2. Let $f \in C[a, b]$. Then for any $\varepsilon>0$, there exists a polynomial $p$ for which $\|f-p\|_{\infty} \leq \varepsilon$.

Proof: See Ref. [13-16].

Theorem 2.3. For any $f \in C[a, b]$ and $N \geq 0$ the best approximation polynomial $p_{N}^{*}(f)$ exists and is unique.

Proof: See Ref. [13-16].

Definition 2.4. Given an integer $N \geq 1$ then a grid set of $(N+1)$ points are $X=\left(x_{i}\right)_{0 \leq i<N}$ in $[a, b]$ such that $a \leq x_{0}<x_{1}<\cdots<x_{N} \leq b$. Then points $\left(x_{i}\right)_{0 \leq i \leq N}$ are called the nodes of the grid.

Theorem 2.5. Given a function $f \in C[a, b]$ and a grid of $(N+1)$ nodes, $X=\left(x_{i}\right)_{0 \leq i \leq N}$, there exists a unique polynomial of degree $N, I_{N}^{X}(f)$ such that

$$
I_{N}^{X}(f)\left(x_{i}\right)=f\left(x_{i}\right), 0 \leq i \leq N
$$

$I_{N}^{X}(f)$ is called the interpolant (or the interpolating polynomial) of $f$ through the grid $X$.

Proof: See Ref. [13-16].

The interpolant $I_{N}^{X}(f)$ can be express in the Lagrange form as

$$
I_{N}^{X}(f)=\sum_{i=0}^{N} f\left(x_{i}\right) \ell_{i}^{X}(x)
$$

where $\ell_{i}^{X}(x)$ is the $\mathrm{i}$-th Lagrance cardinal polynomial associated with the grid $X$ :

$$
\ell_{i}^{X}(x)=\prod_{j=0, i \neq j}^{N} \frac{x-x_{j}}{x_{i}-x_{j}}, 0 \leq i \leq N .
$$

The Lagrange cardinal polynomials are such that

$$
\ell_{i}^{X}\left(x_{j}\right)=\delta_{i j}, 0 \leq i, j \leq N .
$$

The best approximation polynomial $p_{N}^{*}(f)$ is also an interpolant of $f$ at $(N+1)$ nodes the error is given by formula:

$$
\left\|f-I_{N}^{X}(f)\right\|_{\infty} \leq\left(1+\wedge_{N}(X)\right)\left\|f-p_{N}^{*}(f)\right\|_{\infty}
$$

where $\wedge_{N}(X)$ is the Lebesgue constant relative to the grid $X$,

$$
\wedge_{N}(X):=\max _{x \in[a, b]} \sum_{i=0}^{N}\left|\ell_{i}^{X}(x)\right|
$$

The Lebesgue constant contains all the information on the effects of the choice of $X$ on $\left\|f-I_{N}^{X}(f)\right\|_{\infty}$.

Theorem 2.6. For any choice of the grid $X$, there exist a constant $C>0$ such that

$$
\wedge_{N}(X)>\frac{2}{\pi} \ln (N+1)-C
$$

Proof: See Ref. [13-16].

Definition 2.7. The nodal polynomial associated with the grid is the unique polynomial of degree $(N+1)$ and leading coefficient 1 whose zeroes are the $N+1$ nodes of $X$ :

$$
w_{N+1}^{X}(x)=\prod_{i=0}^{N}\left(x-x_{i}\right)
$$

Theorem 2.8. If $f \in C^{N+1}[a, b]$, then for any grid $X$ of $N+1$ nodes, and for any $x \in[a, b]$, the interpolation error at $x$ is

$$
f(x)-I_{N}^{X}(f)(x)=\frac{f^{(N+1)}(\zeta)}{(N+1) !} w_{N+1}^{X}(x)
$$

where $\zeta=\zeta(x) \in[a, b]$ and $w_{N+1}^{X}(x)$ nodal polynomial associated with the grid $X$.

Proof: See Ref. [13-16].

\subsection{The Shifted Chebyshev Polynomial of the First Kind}

Definition 2.9. The Chebyshev polynomial of the first kind $T_{n}(x)$ is a polynomial in $x$ of degree $n$, defined by the relation [17]

$$
T_{n}(x)=\cos (n \theta) \text { when } x=\cos (\theta) .
$$

If the range of the variable $x$ is the interval $[-1,1]$, the range the corresponding variable $\theta$ can be taken $[0, \pi]$. Since the range $[0,1]$ is quite often more convenient to use than the range $[-1,1]$, we map the independent variable $x$ in $[0,1]$ to the variable $s$ in $[-1,1]$ by the transformation 


$$
s=2 x-1 \text { or } x=\frac{1}{2}(1+s)
$$

and this lead to a shifted Chebyshev polynomial of the first kind $T_{n}^{*}(x)$ of degree $n$ in $x$ on $[0,1]$ given by [17]

$$
T_{n}^{*}(x)=T_{n}(s)=T_{n}(2 x-1) .
$$

Thus we have the polynomials

$$
T_{0}^{*}(x)=1, T_{1}^{*}(x)=2 x-1, T_{2}^{*}(x)=8 x^{2}-8 x+1, \cdots
$$

It is of course possible to defined $T_{n}^{*}(x)$, like $T_{n}(x)$, directly by a trigonometric relation. Indeed, we obtained

$$
T_{n}^{*}(x)=\cos 2 n \theta \text { when } x=\cos ^{2} x .
$$

This relation might alternatively be rewritten, with $\theta$ replace by $\phi / 2$, in the form

$$
T_{n}^{*}(x)=\cos (\phi) \text { when } x=\cos ^{2}\left(\frac{\phi}{2}\right)=\frac{1}{2}(1+\cos (\phi))
$$

Note that the shifted Chebyshev polynomial $T_{n}(x)$ is neither even nor odd and indeed all powers of $x$ from $1=x^{0}$ to $x^{n}$ appear in $T_{n}^{*}(x)$. The leading coefficient of $x^{n}$ in $T_{n}^{*}(x)$ for $n>0$ to be $2^{2 n-1}$. These polynomials have the following properties:

1) $T_{n+1}^{*}(x)$ has exactly $n+1$ real zeroes on the interval $[0,1]$. The $m$-th zero $x_{n, m}$ of $T_{n+1}^{*}(x)$ is located at

$$
x_{n, m}=\frac{1}{2}\left(1+\cos \left(\frac{\left(n-m+\frac{3}{2}\right) \pi}{n}\right)\right)
$$

2) It is well known that the relation between the powers $x^{n}$ and the shifted Chebyshev polynomials $T_{n}^{*}(x)$ is

$$
x^{n}=\frac{1}{2^{2 n-1}} \sum_{k=0}^{n}\left(\begin{array}{c}
2 n \\
k
\end{array}\right) T_{n-k}^{*}(x) .
$$

\subsection{Chebyshev-Gauss Grid}

Definition 2.10. The grid $X=\left(x_{i}\right)_{0 \leq i \leq N}$ such that the $x_{i}$ 's are the $N+1$ zeroes of the Chebyshev polynomial of degree $N+1$ is called the Chebshev-Gauss (CG) grid.

Theorem 2.11. The polynomials of degree $N+1$ and leading coefficient 1 , the unique polynomial which has the smallest uniform norm on $[-1,1]$ is the $(n+1)$-th Chebyshev polynomial divided by $2^{N}$.

Proof: See Ref. [13-16].

\section{Fundamental Matrix Relations}

Let us consider the Equation (1) and find the matrix forms of each term of the equation. We first consider the solution $y_{N}(x)$ and its derivative $y_{N}^{(m)}(x)$ defined by a truncated Chebyshev series. Then we can put series in the matrix form

$$
\begin{aligned}
y_{N}(x) & =T_{N}^{*}(x) A, y_{N}^{(1)}(x)=T_{N}^{*(1)}(x) A, y_{N}^{(2)}(x) \\
& =T_{N}^{*(2)}(x) A
\end{aligned}
$$

where

$$
\begin{gathered}
T_{N}^{*}(x)=\left[\begin{array}{llll}
T_{0}^{*}(x) & T_{1}^{*}(x) & \cdots & T_{N}^{*}(x)
\end{array}\right] \\
T_{(N)}^{*(1)}(x)=\left[\begin{array}{llll}
T_{0}^{*(1)}(x) & T_{1}^{*(1)}(x) & \cdots & T_{N}^{*(1)}(x)
\end{array}\right] \\
T_{(N)}^{*(2)}(x)=\left[\begin{array}{llll}
T_{0}^{*(2)}(x) & T_{1}^{*(2)}(x) & \cdots & T_{N}^{*(2)}(x)
\end{array}\right] \\
A=\left[\begin{array}{llll}
a_{0} & a_{1} & \cdots & a_{N}
\end{array}\right]^{T}
\end{gathered}
$$

By using (5), we obtained the corresponding matrix relation as follows:

$$
X^{T}(x)=D T_{N}^{* T}(x) \text { and so } T_{N}^{*}(x)=X(x)\left(D^{T}\right)^{-1}
$$

where

$$
X(x)=\left[\begin{array}{llll}
1 & x & \cdots & x^{N}
\end{array}\right]
$$

and 
Moreover it is clearly seen that the relation between the matrix $X(x)$ and its derivative $X^{(k)}(x)$,

$$
X^{(1)}(x)=X(x) B^{1}, X^{(2)}(x)=X(x) B^{2}
$$

where

$$
B=\left[\begin{array}{ccccc}
0 & 1 & 0 & \cdots & 0 \\
0 & 0 & 2 & \cdots & 0 \\
\vdots & \vdots & \vdots & \ddots & \vdots \\
0 & 0 & 0 & \cdots & N \\
0 & 0 & 0 & \cdots & 0
\end{array}\right] .
$$

The derivative of the matrix $T_{N}^{*}(x)$ defined in (7), by using the relation (8), can expressed as

$$
T_{N}^{*(k)}(x)=X^{(k)}(x)\left(D^{T}\right)^{-1}=X(x) B^{k}\left(D^{T}\right)^{-1}, k=0,1,2(9)
$$

where $T_{N}^{*(0)}(x)=T_{N}^{*}(x), \quad X^{(0)}(x)=X(x), \quad B^{0}=B$. Вy substituting (9) into (6), we obtain

$$
y_{N}^{(k)}(x)=X(x) B^{k}\left(D^{T}\right)^{-1} A, k=0,1,2
$$

where $y_{N}^{(0)}(x)=y_{N}(x)$.

Moreover, we know that;

$$
X(x-\delta)=X(x) B_{\delta}
$$

where

$$
B_{\delta}=\left[\begin{array}{ccccc}
\left(\begin{array}{l}
0 \\
0
\end{array}\right)(-\delta)^{0} & \left(\begin{array}{l}
1 \\
1
\end{array}\right)(-\delta)^{1} & \left(\begin{array}{l}
2 \\
2
\end{array}\right)(-\delta)^{2} & \ldots & \left(\begin{array}{c}
N \\
N
\end{array}\right)(-\delta)^{N} \\
0 & \left(\begin{array}{l}
1 \\
0
\end{array}\right)\left(-\delta^{0}\right) & \left(\begin{array}{l}
2 \\
1
\end{array}\right)(-\delta)^{1} & \ldots & \left(\begin{array}{c}
N \\
N-1
\end{array}\right)(-\delta)^{N-1} \\
0 & 0 & \left(\begin{array}{c}
2 \\
0
\end{array}\right)(-\delta)^{0} & \ldots & \left(\begin{array}{c}
N \\
N-2
\end{array}\right)(-\delta)^{N-2} \\
\vdots & \vdots & \ddots & \vdots \\
0 & 0 & 0 & \ldots & \left(\begin{array}{c}
N \\
0
\end{array}\right)(-\delta)^{0}
\end{array}\right]
$$

Using relation (8) and (11), we can write

$$
X^{(1)}(x-\delta)=X(x) B^{1} B_{\delta}
$$

In a similarly way as (10), we obtain

$$
y^{(1)}(x-\delta)=T_{N}^{*(1)}(x-\delta) A=X(x) B B_{\delta}\left(D^{T}\right)^{-1} A .
$$

\section{Matrix Representation of the Conditions}

Using the relation (10), the matrix form of the conditions given by (2) can be written as

$$
\begin{aligned}
& y(0)=\alpha_{0} \Rightarrow X(0)\left(D^{T}\right)^{-1} A=\left[\alpha_{0}\right] \\
& y(1)=\alpha_{1} \Rightarrow X(1)\left(D^{T}\right)^{-1} A=\left[\alpha_{1}\right]
\end{aligned}
$$

where

$$
\begin{aligned}
& X(0)=\left[\begin{array}{llll}
1 & 0 & \cdots & 0
\end{array}\right] \\
& \left(\varepsilon X\left(x_{i}\right) B^{2}\left(D^{T}\right)^{-1}+p\left(x_{i}\right) X\left(x_{i}\right) B B_{\delta}\left(D^{T}\right)^{-1}+q\left(x_{i}\right) X\left(x_{i}\right)\left(D^{T}\right)^{-1}\right) A=g\left(x_{i}\right)
\end{aligned}
$$

So, the fundamental matrix equation is gained

$$
\left(E X B^{2}\left(D^{T}\right)^{-1}+P X B B_{\delta}\left(D^{T}\right)^{-1}+Q X\left(D^{T}\right)^{-1}\right) A=G
$$

where 


$$
\begin{gathered}
\boldsymbol{P}=\left[\begin{array}{ccccc}
p\left(x_{0}\right) & 0 & 0 & \cdots & 0 \\
0 & p\left(x_{1}\right) & 0 & \cdots & 0 \\
0 & 0 & p\left(x_{2}\right) & \cdots & 0 \\
\vdots & \vdots & \vdots & \ddots & \vdots \\
0 & 0 & 0 & \cdots & p\left(x_{N}\right)
\end{array}\right] \quad \boldsymbol{Q}=\left[\begin{array}{ccccc}
q\left(x_{0}\right) & 0 & 0 & \cdots & 0 \\
0 & q\left(x_{1}\right) & 0 & \cdots & 0 \\
0 & 0 & q\left(x_{2}\right) & \cdots & 0 \\
\vdots & \vdots & \vdots & \ddots & \vdots \\
0 & 0 & 0 & \cdots & q\left(x_{N}\right)
\end{array}\right] \\
X=\left[\begin{array}{ccccc}
1 & x_{0} & x_{0}^{2} & \cdots & x_{0}^{N} \\
1 & x_{1} & x_{1}^{2} & \cdots & x_{1}^{N} \\
1 & x_{2} & x_{2}^{2} & \cdots & x_{2}^{N} \\
\vdots & \vdots & \vdots & \ddots & \vdots \\
1 & x_{N} & x_{N}^{2} & \cdots & x_{N}^{N}
\end{array}\right] \quad G=\left[\begin{array}{c}
g\left(x_{0}\right) \\
g\left(x_{1}\right) \\
g\left(x_{2}\right) \\
\vdots \\
g\left(x_{N}\right)
\end{array}\right] \quad E=\left[\begin{array}{ccccc}
\varepsilon & 0 & 0 & \cdots & 0 \\
0 & \varepsilon & 0 & \cdots & 0 \\
0 & 0 & \varepsilon & \cdots & 0 \\
\vdots & \vdots & \vdots & \ddots & \vdots \\
0 & 0 & 0 & \cdots & \varepsilon
\end{array}\right]
\end{gathered}
$$

The fundamental matrix equation (16) for Equation(1) corresponds to a system of $(N+1)$ algebraic equation for the $(N+1)$ unknown coefficients $a_{0}, a_{1}, \cdots, a_{N}$. Briefly we can write Equation(15) as

$$
W A=G \text { or }[W ; G]
$$

so that, for $p, q=0,1, \cdots, N$

$$
\begin{aligned}
W & =\left[w_{p q}\right] \\
& =E X B^{2}\left(D^{T}\right)^{-1}+P X B B_{\delta}\left(D^{T}\right)^{-1}+Q X\left(D^{T}\right)^{-1}
\end{aligned}
$$

Briefly, the matrix form for conditions (2) are

$$
C_{i} A=\left[\alpha_{i}\right] \text { or }\left[C_{i} ; \alpha_{i}\right], i=0,1
$$

where

$$
C_{i}=X(i)\left(D^{T}\right)^{-1} \equiv\left[\begin{array}{llll}
c_{i 0} & c_{i 1} & \cdots & c_{i N}
\end{array}\right]
$$

To obtain the solution of Equation (1) under the conditions (2), by replacing the rows matrices (18) by the last $\mathrm{m}$ rows of the matrix (17), we have the required augmented matrix

$$
\left[W^{*} ; G^{*}\right]=\left[\begin{array}{cccccc}
w_{00} & w_{10} & \cdots & w_{0 N} & ; & g\left(x_{0}\right) \\
w_{10} & w_{11} & \cdots & w_{1 N} & ; & g\left(x_{1}\right) \\
\vdots & \vdots & \cdots & \vdots & \vdots & \vdots \\
w_{(N-2) 0} & w_{(N-2) 1} & \cdots & w_{(N-2) N} & ; & g\left(x_{N-2}\right) \\
c_{00} & c_{01} & \cdots & c_{0 N} & ; & \alpha_{0} \\
c_{10} & c_{11} & \cdots & c_{1 N} & ; & \alpha_{1}
\end{array}\right]
$$

or corresponding matrix equation

$$
W^{*} A=G^{*}
$$

If $\operatorname{rank}\left(W^{*}\right)=\operatorname{rank}\left[W^{*} ; G^{*}\right]=N+1$, then we can write

$$
A=\left(W^{*}\right)^{-1} G^{*} .
$$

Thus the coefficients $a_{i}, i=0, \cdots, N$ are uniquely determined by Equation (20).

\subsection{Convergence and Error Analysis}

Since $\left\|T_{N+1}^{*}\right\|_{\infty}=1$, we conclude that if we choose the grid nodes $\left(x_{i}\right)_{0 \leq i \leq N}$ to be zero the $(N+1)$ zeroes of the shifted Chebyshev polynomial $T_{N+1}^{*}$, we have

$$
\left\|w_{N+1}^{X}\right\|_{\infty}=\frac{1}{2^{2 N-1}}
$$

and this is the smallest possible value. In particular, for any $f \in C^{N+1}[0,1]$ we have [14]

$$
\left\|y-y_{N}\right\|_{\infty} \leq \frac{1}{2^{2 N-1}(N+1) !}\left\|y^{(N+1)}\right\|_{\infty}
$$

If $y^{(N+1)}$ is uniformly bounded, the convergence of the interpolation $y_{N}$ towards $y(x)$ when $N \rightarrow \infty$ is then extremely fast. Also the Lebesgue constant associated with the Chebyshev-Gauss grid is small

$$
\wedge_{N}(X) \sim \frac{2}{\pi} \ln (N+1) \text { as } N \rightarrow \infty .
$$

This is much better than uniform grids and close to the optimal value.

\subsection{Checking of Solution}

We can easily check the accuracy of the obtained solutions as follows: Since the obtained the shifted Chebyshev polynomial of the first kind expansion is an approximate solution of Equation (1), when the function $y_{N}(x)$ and its derivatives are substituted in Equation (1), the resulting equation must be satisfied approximately, that is for $x_{i} \in[0,1]$

$$
\begin{aligned}
& E_{\varepsilon, \delta}(N)= \\
& \left|\varepsilon y^{\prime \prime}\left(x_{i}\right)+p\left(x_{i}\right) y^{\prime}\left(x_{i}-\delta\right)+q\left(x_{i}\right) y\left(x_{i}\right)-g\left(x_{i}\right)\right| \cong 0
\end{aligned}
$$

\section{Illustrative Example}

To demonstrate the effect of delay on the layer behavior 
of the solution and the efficiency of the method, we consider the examples given below and solve them using the present method and all of them were performed on the computer using a program written in Maple 9 software in the solving process. We have plotted the graphs of the solution of the problem for different $\varepsilon$ with different values of $\delta$ to show the effect of delay on the boundary layer solution.

The maximum errors denoted by $E_{N, \varepsilon}$ at all the grid points are evaluated using the formula

$$
E_{N, \varepsilon}=\max _{0 \leq i \leq N}\left|y\left(x_{i}\right)-y_{N}\left(x_{i}\right)\right|
$$

Example 5.1. Let us consider the second-order singu-

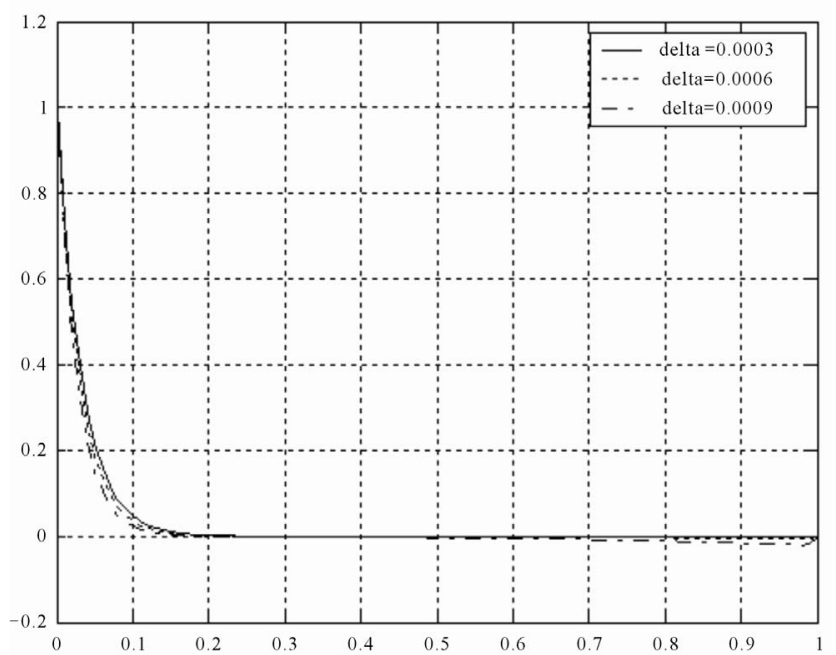

(a)

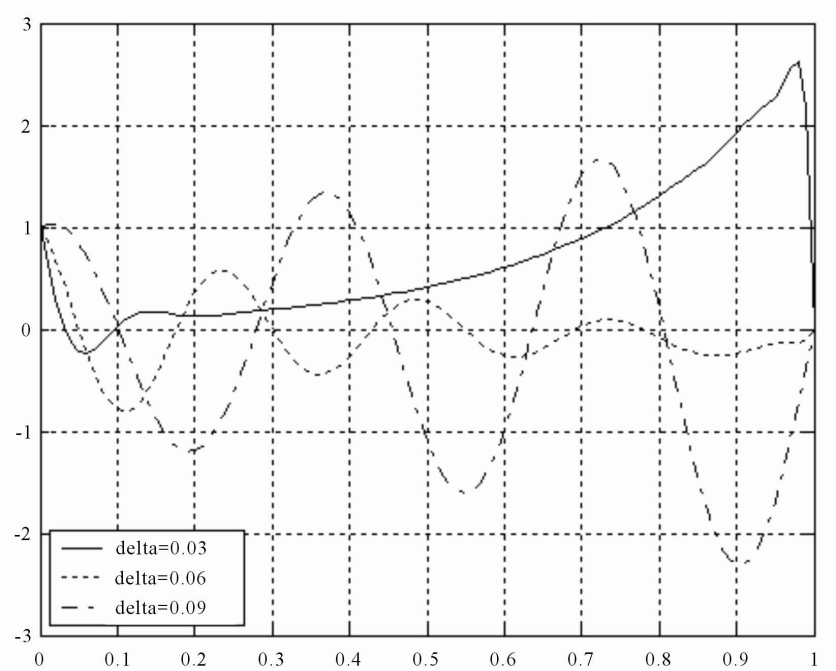

(c) larly-perturbed delay differential equation [3]

$$
\varepsilon y^{\prime \prime}-y+0.25 y^{\prime}(x-\delta)=0
$$

under the conditions $y(0)=1, y(1)=0$. The exact solution for the considered examples is not available but we compare numerical results in Figures 1(a) and (b) given by Kadalbajoo ([3], Example 2). In Figure 1(a), we show the numerical result using present method and for the method using by Kadalbajoo is shown in Figure 1(b). The graphs of the solution of the considered examples for different values of delay are plotted in Figures 1(a)-(d) to examine the questions on the effect of delay on the boundary layer behavior of the solution.

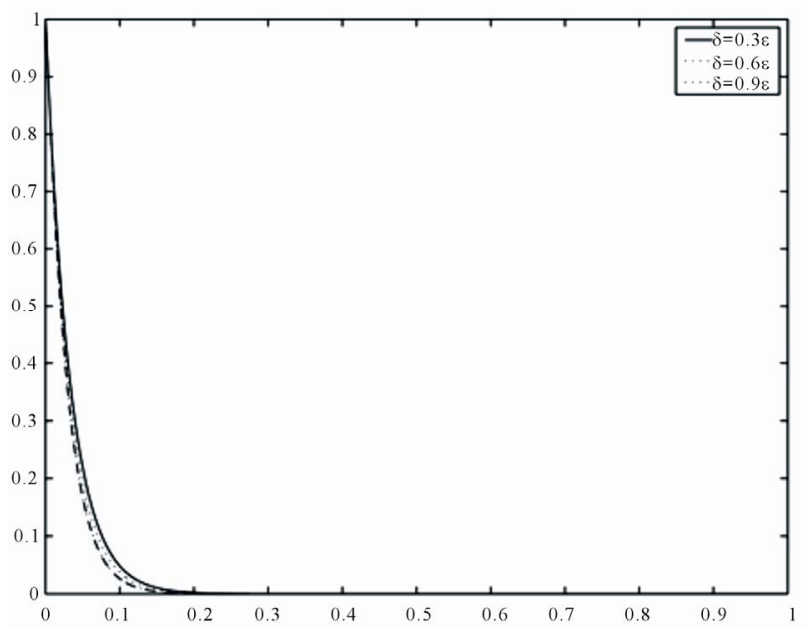

(b)

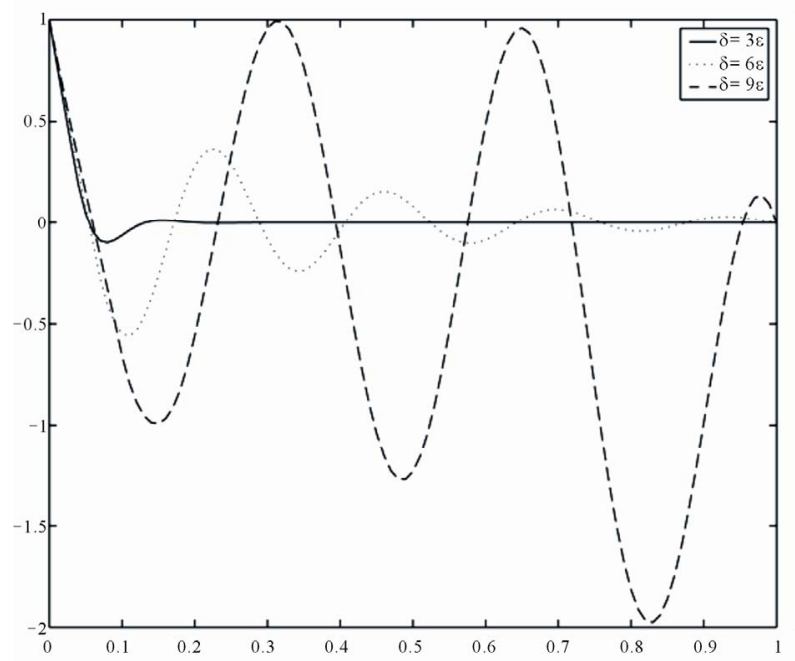

(d)

Figure 1. (a) Numerical results of Example 5.1 for various $\delta(\varepsilon=0.01, N=20)$; (b) Numerical results of Example 5.1 for various $\delta$ in [3] $(\varepsilon=\mathbf{0 . 0 1})$; (c) Numerical results of Example 5.1 for various $\delta(\varepsilon=0.01, N=20)$; (d) Numerical results of Example 5.1 for various $\delta$ in [3] $(\varepsilon=0.01)$. 
Example 5.2. Let us consider the second-order singularly-perturbed delay differential equation [3]

$$
\varepsilon y^{\prime \prime}-y+y^{\prime}(x-\delta)=0
$$

under the conditions $y(0)=1, y(1)=1$. Its exact solution is given by

$$
y(x)=\frac{\left(1-e^{m_{2}}\right) e^{m_{1} x}+\left(e^{m_{1}}-1\right) e^{m_{2} x}}{e^{m_{1}}-e^{m_{2}}}
$$

where

$$
\begin{aligned}
& m_{1}=\frac{-1-\sqrt{1+4(\varepsilon-\delta)}}{2(\varepsilon-\delta)} \\
& m_{1}=\frac{-1+\sqrt{1+4(\varepsilon-\delta)}}{2(\varepsilon-\delta)} .
\end{aligned}
$$

We solved this problem using the method presented here and compared the result with the exact solution of the problems in Table 1. Also for $N=10$, we have plotted the graphs of the computed solution of the problem for $\varepsilon=2^{-2}$, for different values $\delta$ in Figure 2(a) and we can easily check the accuracy of the obtained solutions in Figure 2(b).

Example 5.3. Let us consider the second-order singularly-perturbed delay differential Equation [6]

$$
\varepsilon y^{\prime \prime}-y-y^{\prime}(x-\delta)=0
$$

under the conditions $y(0)=1, y(1)=-1$. Its exact so- lution is given by

$$
y(x)=\frac{-\left(1+e^{m_{2}}\right) e^{m_{1} x}+\left(e^{m_{1}}+1\right) e^{m_{2} x}}{e^{m_{1}}-e^{m_{2}}}
$$

where

$$
\begin{aligned}
& m_{1}=\frac{1+\sqrt{1+4(\varepsilon+\delta)}}{2(\varepsilon+\delta)} \\
& m_{1}=\frac{1-\sqrt{1+4(\varepsilon+\delta)}}{2(\varepsilon+\delta)} .
\end{aligned}
$$

We calculated numerical results for $\delta=0.5 \varepsilon$ and display results for some various $N$. Moreover, we compare the results with Non-standard finite difference methods (NSFDMs) in Table 2 and we display results for $N=10$, in Figure 3 .

Table 1. $E_{N, 2^{-2}}$ values of Example 5.1.

\begin{tabular}{cccc}
\hline$\delta$ & $N=10$ & $N=30$ & $E_{2^{-2}, \delta}(10)$ \\
\hline $2^{-8}$ & $0.315 \mathrm{E}-4$ & $0.252 \mathrm{E}-4$ & $0.426 \mathrm{E}-2$ \\
$2^{-10}$ & $0.138 \mathrm{E}-4$ & $0.153 \mathrm{E}-5$ & $0.392 \mathrm{E}-2$ \\
$2^{-12}$ & $0.134 \mathrm{E}-4$ & $0.935 \mathrm{E}-7$ & $0.384 \mathrm{E}-2$ \\
$2^{-14}$ & $0.133 \mathrm{E}-4$ & $0.594 \mathrm{E}-8$ & $0.382 \mathrm{E}-2$ \\
\hline
\end{tabular}

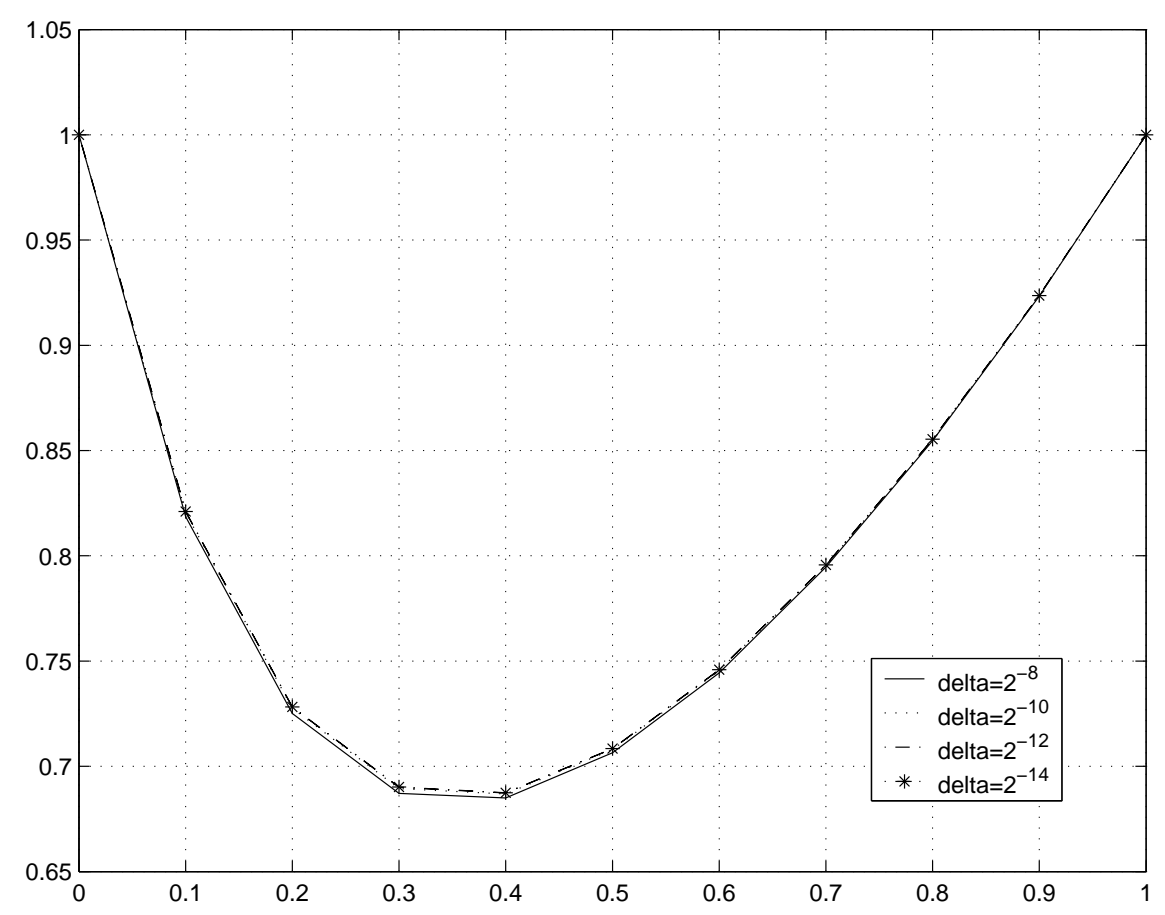

(a) 


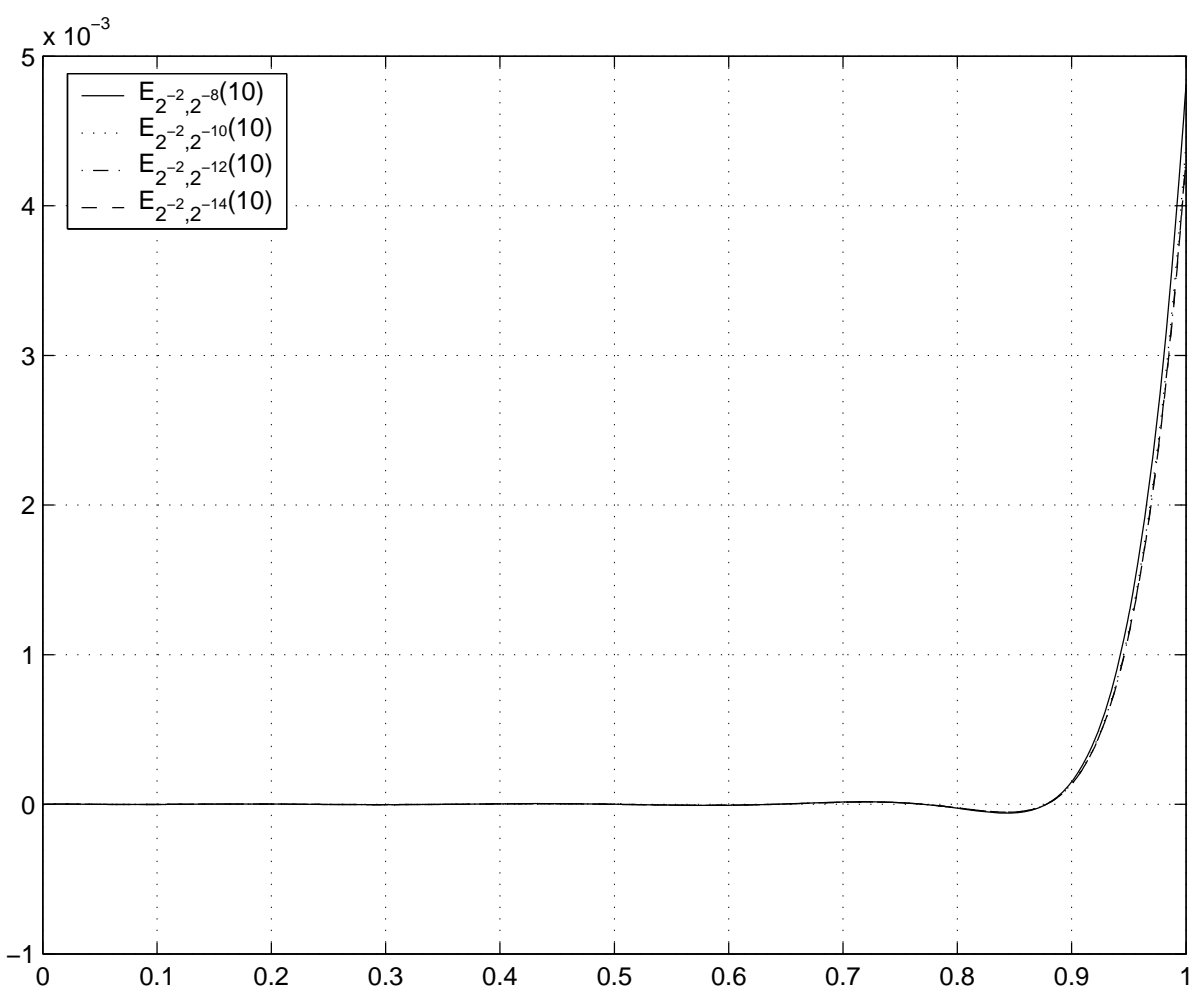

(b)

Figure 2. (a) Numerical results of Example 5.2 for various $\delta(N=10)$; (b) For $E_{2^{-2}, \delta}(10)$, numerical results of Example 5.2 for various $\delta$.

Table 2. For $\delta=0.5 \varepsilon, \quad E_{N, \varepsilon}$ values of Example 5.3.

\begin{tabular}{ccccccc}
\hline$\varepsilon$ & $N=10$ & $N=20$ & $N=30$ & $N=40$ & NFSDs $(N=32)$ & NFSDs $(N=64)$ \\
\hline $2^{-6}$ & $0.213 \mathrm{E}-0$ & $0.222 \mathrm{E}-1$ & $0.258 \mathrm{E}-1$ & $0.201 \mathrm{E}-1$ & $0.340 \mathrm{E}-1$ & $0.170 \mathrm{E}-1$ \\
$2^{-7}$ & $0.311 \mathrm{E}-0$ & $0.538 \mathrm{E}-1$ & $0.347 \mathrm{E}-1$ & $0.265 \mathrm{E}-1$ & $0.340 \mathrm{E}-1$ & $0.170 \mathrm{E}-1$ \\
$2^{-8}$ & $0.613 \mathrm{E}-0$ & $0.224 \mathrm{E}-0$ & $0.336 \mathrm{E}-1$ & $0.218 \mathrm{E}-1$ & $0.340 \mathrm{E}-1$ & $0.170 \mathrm{E}-1$ \\
$2^{-9}$ & $0.941 \mathrm{E}-0$ & $0.340 \mathrm{E}-0$ & $0.202 \mathrm{E}-0$ & $0.598 \mathrm{E}-1$ & $0.340 \mathrm{E}-1$ & $0.170 \mathrm{E}-1$ \\
$2^{-10}$ & $1.136 \mathrm{E}-0$ & $0.656 \mathrm{E}-0$ & $0.306 \mathrm{E}-0$ & $0.232 \mathrm{E}-0$ & $0.340 \mathrm{E}-1$ & $0.170 \mathrm{E}-1$ \\
\hline
\end{tabular}

\section{Conclusions}

In recent years, the studies of the singularly-perturbed delay differential equations have attracted the attention of many sciences and engineers. The Chebyshev expansion methods are used to solve the singularly-perturbed delay differential equations numerically. A considerable advantage of the method is that the Chebyshev polynomial coefficients of the solution are found very easily by using computer programs in Maple 9. Shorter computation time and lower operation count results in reduction of cumulative truncation errors and improvement of overall accuracy. Illustrative examples are included to demonstrate the validity and applicability of the technique. To get the best approximating solution of the equation, we take more forms from the Chebyshev expansion of functions, that is, the truncation limit $\mathrm{N}$ must be chosen large enough. Suggested approximations make this method very attractive and contributed to the good agreement between approximate and exact values in the numerical example.

As a result, the power of the employed method is confirmed. We assured the correctness of the obtained solutions by putting them back into the original equation with 


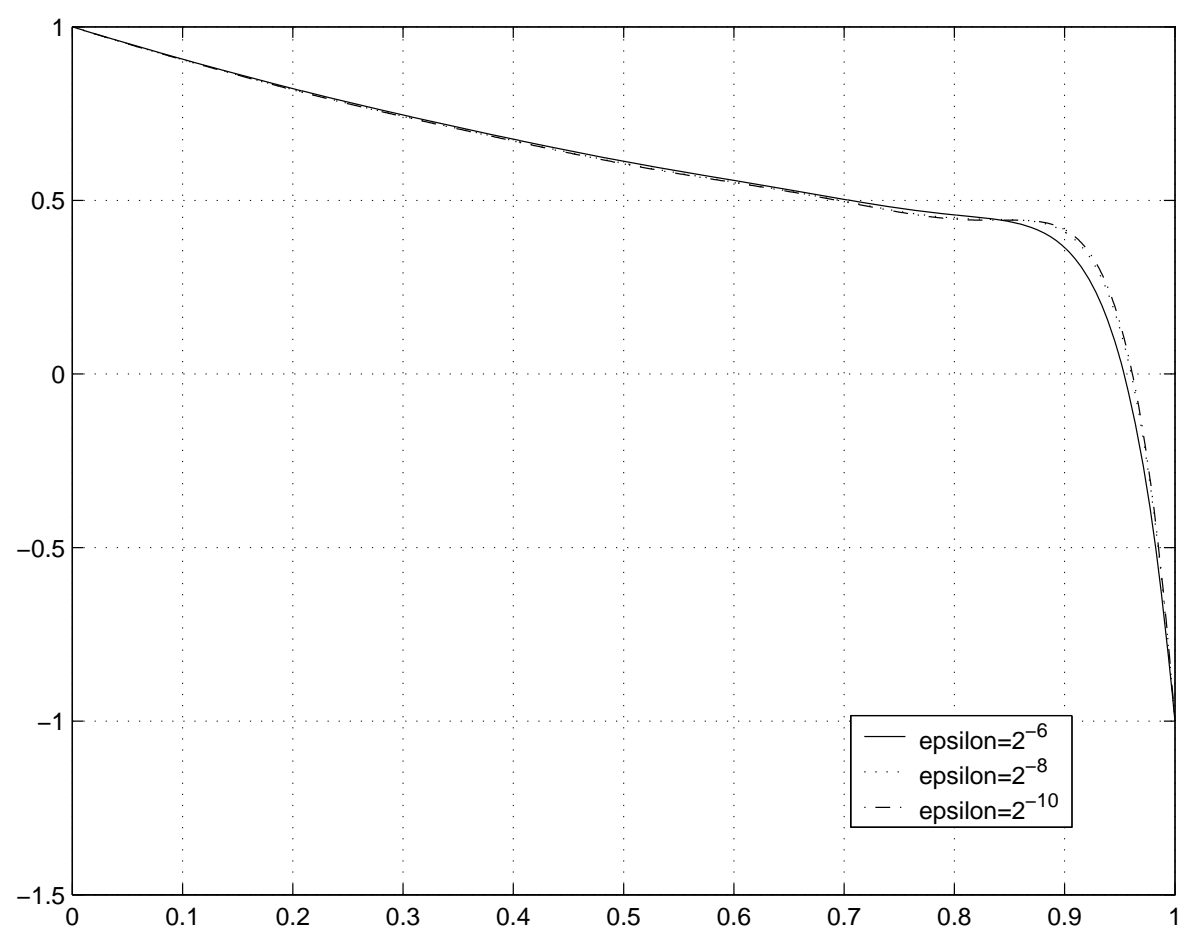

Figure 3. Numerical results of Example 5.3 for various $\delta$.

the aid of Maple, it provides an extra measure of confidence in the results.

\section{References}

[1] M. K. Kadalbajoo and D. Kumar, "Fitted Mesh $B$-Spline Collocation Method for Singularly Perturbed DifferentialDifference Equations with Small Delay," Applied Mathematics and Computation, Vol. 204, No. 1, 2008, pp. 9098. doi:10.1016/j.amc.2008.05.140

[2] M. K. Kadalbajoo and K. K. Sharma, "A Numerical Method Based on Finite Difference for Boundary Value Problems for Singularly Perturbed Delay Differential Equations," Applied Mathematics and Computation, Vol. 197, No. 2, 2008, pp. 692-707. doi:10.1016/j.amc.2007.08.089

[3] M. K. Kadalbajoo and V. P. Ramesh, "Numerical Methods on Shishkin Mesh for Singularly Perturbed Delay Differential Equations with a Grid Adaptation Strategy," Applied Mathematics and Computation, Vol. 188, No. 2, 2007, pp. 1816-1831. doi:10.1016/j.amc.2006.11.046

[4] M. K. Kadalbajoo and V. P. Ramesh, "Hybrid Method for Numerical Solution of Singularly Perturbed Delay Differential Equationsy," Applied Mathematics and Computation, Vol. 187, No. 2, 2007, pp. 797-814. doi:10.1016/j.amc.2006.08.159

[5] M. K. Kadalbajoo and K. K. Sharma, "Numerical Analysis of Singularly Perturbed Delay Differential Equations with Layer Behavior," Applied Mathematics and Computation, Vol. 157, No. 1, 2004, pp. 11-28. doi:10.1016/j.amc.2003.06.012

[6] K. C. Patidar and K. K. Sharma, " $\varepsilon$-Uniformly Conver- gent Non-Standard Finite Difference Methods for Singularly Perturbed Differential Difference Equations with Small Delay," Applied Mathematics and Computation, Vol. 175, No. 1, 2006, pp. 864-890. doi:10.1016/j.amc.2005.08.006

[7] M. H. Adhikari, E. A. Coutsias and J. K. Mclver, "Periodic Solutions of a Singularly Perturbed Delay Differential Equation," Physica D, Vol. 237, No. 24, 2008, pp. 3307-3321. doi:10.1016/i.physd.2008.07.019

[8] I. G. Amirsliyeva, F. Erdogan and G. M. Amiraliyev, "A Uniform Numerical Method for Dealing with a Singularly Perturbed Delay Initial Value Problem," Applied Mathematics Letters, Vol. 23, No. 10, 2010, pp. 1221-1225. doi:10.1016/j.aml.2010.06.002

[9] S. N. Chow and J. M. Paret, "Singularly Perturbed DelayDifferential Equations, Cuopled Nonlinear Oscillators," North-Holland Publishing Company, Amsterdam, 1983.

[10] R. E. O’Malley Jr., "Introduction to Singular Perturbation," Academic Press, New York, 1979.

[11] M. Glsu, Y. Öztürk and M. Sezer, "A Newcollocation Method for Solutionof the Mixed Linear Integro-Differential-Difference Equations," Applied Mathematics and Computation, Vol. 216, No. 7, 2010, pp. 2183-2198. doi:10.1016/j.amc.2010.03.054

[12] M. Sezer and M. Gulsu, "Polynomial Solution of the Most General Linear Fredholm Integro-Differential-Difference Equation by Means of Taylor Matrix Method," International Journal of Complex Variables, Vol. 50, No. 5, 2005, pp. 367-382.

[13] T. J. Rivlin, "Introduction to the Approximation of Functions," Courier Dover Publications, London, 1969. 
[14] P. J. Davis, "Interpolation and Approximation,” Dover Publications, New York, 1963.

[15] K. Atkinson and W. Han, "Theoretical Numerical Analysis," 3rd Edition, Springer, 2009.
[16] J. P. Body, "Chebyshev and Fourier Spectral Methods," University of Michigan, New York, 2000.

[17] J. C. Mason and D. C. Handscomb, "Chebyshev Polynomials," Chapman and Hall/CRC, New York, 2003. 